\title{
Ultrasound and Fluoroscopic Images Fusion by Autonomous Ultrasound Probe Detection
}

\author{
Peter Mountney ${ }^{1}$, Razvan Ionasec ${ }^{1}$, Markus Kaizer ${ }^{2}$, Sina Mamaghani ${ }^{1}$, Wen Wu ${ }^{1}$, \\ Terrence Chen ${ }^{1}$, Matthias John ${ }^{2}$, Jan Boese ${ }^{2}$, and Dorin Comaniciu ${ }^{1}$ \\ ${ }^{1}$ Siemens Corporate Research \& Technology, Princeton, USA \\ ${ }^{2}$ Siemens AG, Healthcare Sector, Forchheim, Germany
}

\begin{abstract}
New minimal-invasive interventions such as transcatheter valve procedures exploit multiple imaging modalities to guide tools (fluoroscopy) and visualize soft tissue (transesophageal echocardiography (TEE)). Currently, these complementary modalities are visualized in separate coordinate systems and on separate monitors creating a challenging clinical workflow. This paper proposes a novel framework for fusing TEE and fluoroscopy by detecting the pose of the TEE probe in the fluoroscopic image. Probe pose detection is challenging in fluoroscopy and conventional computer vision techniques are not well suited. Current research requires manual initialization or the addition of fiducials. The main contribution of this paper is autonomous six DoF pose detection by combining discriminative learning techniques with a fast binary template library. The pose estimation problem is reformulated to incrementally detect pose parameters by exploiting natural invariances in the image. The theoretical contribution of this paper is validated on synthetic, phantom and in vivo data. The practical application of this technique is supported by accurate results $(<5 \mathrm{~mm}$ in-plane error) and computation time of $0.5 \mathrm{~s}$.
\end{abstract}

\section{Introduction}

Percutaneous and minimally-invasive cardiac procedures are progressively replacing conventional open-heart surgery for the treatment of structural and rhythmological heart disease. Catheters are used to access target anatomy through small vascular access ports. This greatly reduces recovery time and the risk of complications associated with open surgery. Without direct access and visualization, the entire procedure is performed under imaging guidance. There are two established modalities currently used in operating rooms to provide real-time intra-operative images: X-ray fluoroscopy (Fluoro) and transesophageal echocardiography (TEE). Fluoro provides high quality visualization of instruments and devices, which are typically radiopaque, while TEE and more recently 3D TEE can image soft-tissue with great detail. Nevertheless, the complementary nature of TEE and Fluoro is barely exploited in today's practice where the real-time acquisitions are not synchronized and images are visualized separately in misaligned coordinate systems.

Recently, the fusion of Fluoro and TEE has been proposed using either hardware or image based methods. Hardware based approaches [1],[2] attach additional devices to 
the ultrasound probe such as electromagnetic [1] or mechanical [2] trackers and align the device and Fluoro coordinates systems through calibration. Image based methods $[3],[4],[5]$ attempt to use the appearance of the TEE probe in the Fluoro image to estimate the pose of the probe in the Fluoro coordinate system. These methods are attractive because they do not require the introduction of additional equipment into the theatre which may disrupt clinical workflow.

Image based pose estimation is well studied and the problem may be considered solved when the correspondence between 2D image points and a 3D model are known. Unfortunately, the appearance of the TEE probe in the Fluoro image makes establishing the correspondence challenging. The probe's appearance lacks texture or clear feature points and can be homogenous under low dose or close to dense tissue. To alleviate this problem, markers [5] may be retro fitted to the TEE probe. The pose of the probe is estimated using well established computer vision techniques, however, the addition of markers increases the overall size of the probe. Alternatively the natural geometry of the probe may be used to estimate its pose [3],[4]. The authors use a $2 \mathrm{D} / 3 \mathrm{D}$ registration technique to refine the probe's pose estimation and optimal results are obtained using two biplane images. The method is robust for small pose changes $\left(10 \mathrm{~mm} / 10^{\circ}\right)$, however, it requires manual initialization and does not update the registration in real-time, both of which are important in the clinical setting.

In the paper we propose a robust and fast learning-based method for the automated detection of the TEE probe pose, with six degrees of freedom, from Fluoro images. A probabilistic model-based approach is employed to estimate candidates for the inplane probe position, orientation and scale parameters. Digitally reconstructed radiography (DRR) in combination with a binary template library is introduced for the estimation of out-of-plane rotation parameters (pitch and roll). The approach does not require manual initialization, is robust over the entire pose parameter space, and independent of specific TEE probe design / manufacturer. The performance of the algorithm is demonstrated on a comprehensive dataset of in vivo Fluoro sequences and validated on simulated and phantom data.

\section{Fusion Framework}

Information from a TEE volume can be visualized in a Fluoro image by aligning the TEE and C-arm Fluoro coordinate systems. A point in the ultrasound volume $Q^{T E E}$ can be visualized in the Fluoro image at coordinate $Q^{\text {Fluoro }}$ using the following transformation

$$
Q^{\text {Fluorolmage }}=P_{\text {projection }} R_{x z} T_{d} R_{\gamma} R_{\alpha}\left(R_{T E E}^{W} Q^{T E E}+T_{T E E}^{W}\right)
$$

where $P$ projection is the projection matrix, $R_{x z}$ and $T_{d}$ are the transformation from detector to world coordinate system, $R_{\gamma}$ and $R \alpha$ are the angulations of the C-arm and $R_{T E E}^{W}$ and $T_{T E E}^{W}$ are the rotation and position of the TEE probe in the world coordinate system such that $R_{T E E}^{W}=R_{\alpha}^{-1} R_{\gamma}^{-1} R_{x z}^{-1} R_{T E E}^{\text {FluoroDetector }}$ and $T_{T E E}^{W}=R_{\alpha}^{-1} R_{\gamma}^{-1} T_{d}^{-1} R_{x z}^{-1} R_{T E E}^{\text {FluoroDetector }}$. 
The TEE volume and Fluoro image can be aligned if position $T_{\text {TEE }}^{\text {Fluorotector }}=(x, y, z)$ and orientation $R_{T E E}^{\text {FluoroDetector }}=(\theta r, \theta p, \theta y)$ of the TEE probe are known in the Fluoro detector coordinate system.

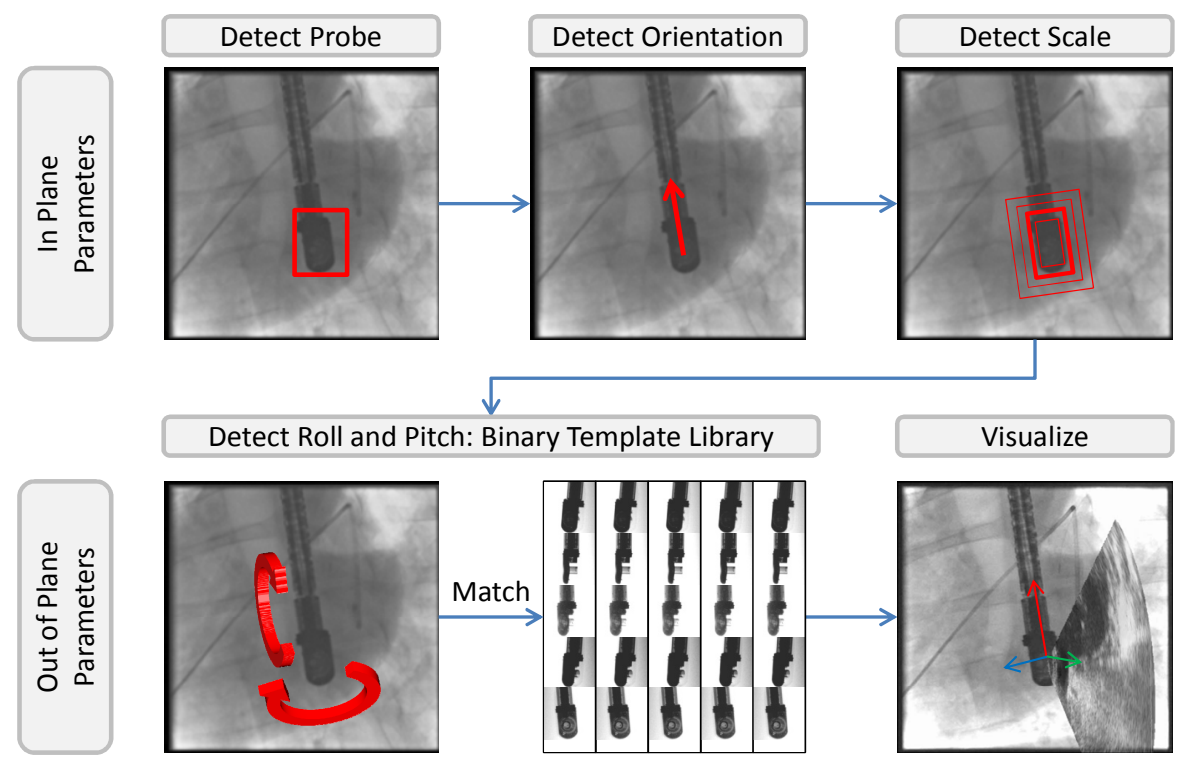

Fig. 1. Detecting the pose of a TEE probe from a single Fluoro image

\subsection{TEE Probe Pose Detection}

At the heart of our approach is the separation of the pose parameters into in-plane $(x, y, z)$ and $(\theta y)$ and out-of-plane $(\theta r, \theta p)$ parameters (shown in Fig. 1). By marginalizing the estimation problem, in-plane parameters can be efficiently estimated directly from the Fluoro images $I^{\text {Fluoro }}$, while being invariant to the out-ofplane parameters that are more challenging to determine.

The in-plane parameters can be computed from the probe's position $(u, v)$, size $(s)$ and orientation $(\theta y)$ in the Fluoro image, the projection transformation Projection of the Fluoro device and the physical dimensions of the TEE probe. To detect the in-plane parameters $(u, v),(s),(\theta y)$ from a Fluoro image $I^{\text {Fluoro }}$ we use discriminative learning methods as described in the next section.

The out-of-plane parameters are more challenging to estimate. The visual appearance in Fluoro of the probe varies greatly making it challenging to learn a compact classifier. This requires the problem to be treated in a fundamentally different way. A template library is created of the probe's appearance under out-of-plane orientations $(\theta r, \theta p)$. Each template has an associated $(\theta r, \theta p)$ and by matching the Fluoro image to the template the out-of-plane parameters can be estimated. 


\section{Detecting In-plane Parameters}

The in-plane parameters are estimated using discriminative learning methods. A classifier is trained to detect the position $(u, v)$, size $(s)$ and orientation $(\theta y)$ of the TEE probe in the Fluoro image. The classifiers are trained using manually annotated Fluoro data. They are trained and applied sequentially such that first, candidates are detected for $(u, v)$, then the orientation $(\theta y)$ is detected for each candidate and finally the size of the probe is detected $(s)$. Each detector is trained using a Probabilistic Boosting Tree (PBT) with Haar-like and steerable features [6].

The position $(u, v)$ detector is trained on manual annotations and negative examples taken randomly from the Fluoro image. The Fluoro image is resized to $128 \times 128$ and a window of $35 \times 35$ is centered at the annotation. A pool of 100,000 Haar features are used to train the PBT. The appearance of the probe varies greatly and to avoid over fitting a classifier is created which is less discriminative but highly probably to detect the tip of the probe.

The orientation $(\theta y)$ detector is trained on manually annotated data and the false positives from the position detector. Additional negative training data is created, centered on the annotation but with incorrect orientation parameters. The PBT is trained with five features including the relative intensity and the difference between two steerable filters [6]. The orientation detector is trained at intervals of $6^{\circ}$ with $360^{\circ}$ coverage. This detector is more discriminative than the position detector and therefore removes outliers as well as estimating the orientation.

The size $(s)$ detector is trained to detect two points where the tip of the probe meets the shaft. The PBT is trained using Haar features. During detection the orientation and position of the probe are used to constrain the search area for the size detector.

\section{Detecting Out-of-plane Parameters}

The appearance of the probe under roll and pitch $(\theta r, \theta p)$ varies significantly in the Fluoro image and cannot generally be accounted for in the image space using the same techniques as the in-plane parameters. The out-of-plane parameters must be treated in a fundamentally different way. The proposed solution is to build a template library containing Fluoro images of the probe under different $(\theta r, \theta p)$. The $(\theta r, \theta p)$ parameters are estimated by matching an image patch in $I^{\text {Fluoro }}$ (normalized for the in-plane parameters) with the template library.

A comprehensive template library should contain a wide variety of orientations. It is not feasible to build this library from in vivo data as it is challenging to manually annotate $(\theta r, \theta p)$ and the data may not contain complete coverage of the parameter space. The library is constructed using DRR. DRR's simulate x-ray Fluoro by tracing light rays through a $3 \mathrm{D}$ volume. In this work a DynaCT of the TEE probe is acquired $(512 \times 512 \times 4880.2225 \mathrm{~mm}$ resolution). The orientation and position of the probe was manually annotated and $(\theta r, \theta p)$ rotations are applied to the volume.

Searching a large template library can be computationally expensive. The size of the library is limited to reduce the search space. The probe is not free to move in all directions due to the physical constraints of the tissue. In addition the X-ray image, formulated by integrating light, makes objects appear the same under symmetrical poses. This is exploited to reduce the size of the template library. The library is built 
with pitch $(\theta p)$ from $-45^{\circ}$ to $45^{\circ}$ and roll $(\theta r)$ from $-90^{\circ}$ to $90^{\circ}$ at $2^{\circ}$ intervals. This subsample library is still large and expensive to store and search. To make the problem computationally tractable a binary template representation is used [7],[8]. Binary templates are an efficient way of storing discriminative information for fast matching.

The image patch is divided into sub-regions and features are extracted for each region. The dominant orientation [7] of the gradient in the sub-region is used as a feature. This has been shown to work well on homogenous regions and objects which lack texture as is the case for the TEE probe in the Fluoro image. The orientations are discretized into 8 orientation bins. Each sub-region can be represented as a single byte which corresponds to the 8 orientation bins. The bit is set to 1 if the orientation exists in the sub-region and 0 if it does not. The binary template for the image patch is comprised of a set of bytes corresponding to the sub-regions. The resulting template is a compact and discriminative representation of the image patch.

Input templates extracted from the Fluoro image $F\left(I^{\text {Fluoro }}\right)$ are matched to templates in the library $F(O)$ using

$$
\varepsilon_{1}\left(I^{\text {Fluoro }}, O, c\right)=\sum_{r} \delta\left(F\left(I^{\text {Fluoro }},(u, v)+r\right)=F(O, r)\right)
$$

where $\delta()$ is a binary function which returns true if the features in two regions match, $F\left(I^{\text {Fluoro }},(u, v)+r\right)$ is the input template centered on candidate $(u, v)$ in image $I^{\text {Fluoro }}$, $F(O, r)$ is a template in the library and $r$ is the sub-region. The function counts how many sub-regions in two templates are the same. The template in the library with the highest count is taken to be the best match and the associated $(\theta r, \theta p)$ as the out-ofplane parameters. This function can be evaluated very quickly using a bitwise AND operation followed by a bit count enabling the library to be searched efficiently.

\section{Results}

The proposed method for probe pose detection was validated on synthetic, phantom and in vivo datasets. Throughout our experiments a GE TEE Transducer was used. The synthetic dataset includes 4050 simulated Fluoro images (DRR) from a 3D C-arm Volume (DynaCT $-512 \times 512 \times 4880.2225 \mathrm{~mm}$ pixel spacing) of the TEE probe. The groundtruth was generated by annotating the $3 \mathrm{D}$ probe position in the DynaCT volume. The phantom dataset includes a volumetric DynaCT of the TEE probe inserted into a silicon phantom, and a total of 51 Fluoro ( $960 \times 9600.184 \mathrm{~mm}$ pixel spacing) images captured by rotating the $\mathrm{C}$-arm and with the TEE probe remaining static.

The position of the C-arm is known from the robotic control, which enabled groundtruth to be computed for each Fluoro image using the 3D probe annotation. The in vivo dataset was acquired during several porcine studies and includes 50 Fluoro sequences comprising of around 7,000 frames ( $512 \times 5120.345 \mathrm{~mm}$ pixel spacing). The data contains images with background clutter, catheter tools and variety in the pose of the probe, C-arm angulations, dose and anatomy. The pose parameters were manually annotated in all frames and assumed as ground-truth for training and testing. 

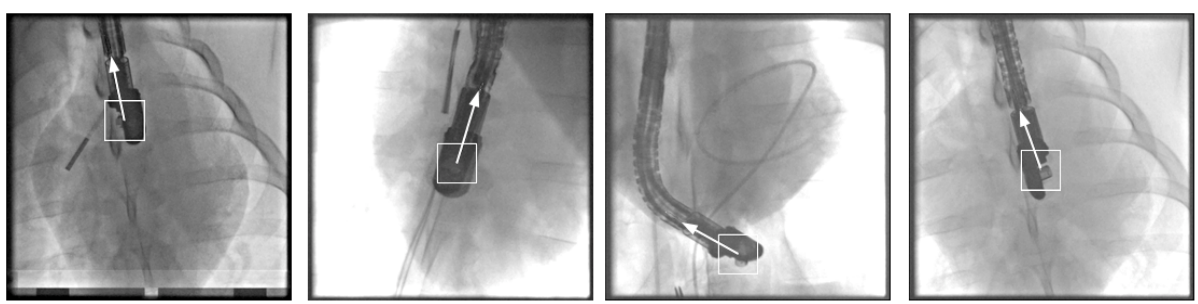

Fig. 2. Fluoroscopic images illustrating probe detection and estimation of in-plane parameters

In the first experiment the quantitative and qualitative performance evaluation of the in-plane parameter $\left(u, v, \theta_{y}\right)$ detection was performed on all three datasets. The detector was trained on $75 \%$ of the in vivo dataset (36 sequences of 5,363 frames) and tested on the entire synthetic, phantom and remaining $25 \%$ of the in vivo dataset. The results are summarized in Table 1.

For the in vivo data the average in-plane position $(u, v)$ error was 2.2 and $3.7 \mathrm{~mm}$ and the in-plane orientation error was $6.69^{\circ}$. Errors in the position estimation are caused by false detections along the shaft of the probe. False position detections contribute to errors in the orientation estimation. The true positive rate is 0.88 and the false positive rate is 0.22 . The detection and accuracy is affected by dose level, proximity to dense tissue and background clutter. The detection framework performs best when the probe is clearly distinguishable from its background. Fig. 2 illustrates detection examples and nature of in vivo images with cluttered background and low textured probe.

The results for the phantom and synthetic data are provided in Table 1 where detection was performed at a fixed scale. The Fluoro data from the phantom experiment appears different from the in vivo data used to train the detectors making it challenging. The true positive rate was 0.95 and false positive rate 0.05 . False detections were caused by the density of the silicon phantom, which obscures the probe in three images. The true positive and false positive rates for synthetic data were 0.99 and 0.01 respectively. The visual appearance of the synthetic DRR is similar to the training data and the probe is clearly distinguishable causing high true positive rate.

Table 1. Quantitative validation of the in-plane position $(u, v)$ and orientation $(\theta y)$.

\begin{tabular}{|c|c|c|c|}
\hline & \multicolumn{3}{|c|}{ Average Error } \\
\hline Data & $\mathrm{u}(\mathrm{mm})$ & $\mathrm{v}(\mathrm{mm})$ & $(\theta y)$ \\
\hline Synthetic & $1.1(1.1)$ & $2.2(3.9)$ & $2.6(3.2)$ \\
\hline Phantom & $1.6(1.4)$ & $2.0(1.2)$ & $3.0(3.4)$ \\
\hline In Vivo & $2.2(5.1)$ & $3.7(8.0)$ & $6.6(16.7)$ \\
\hline
\end{tabular}

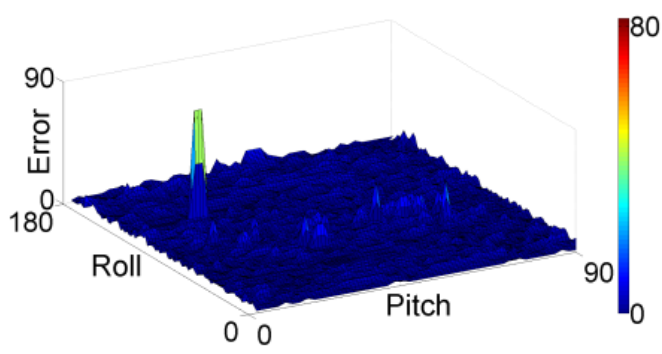

Fig. 3. Error analysis (degrees) of $(\theta r, \theta p)$ over search space 

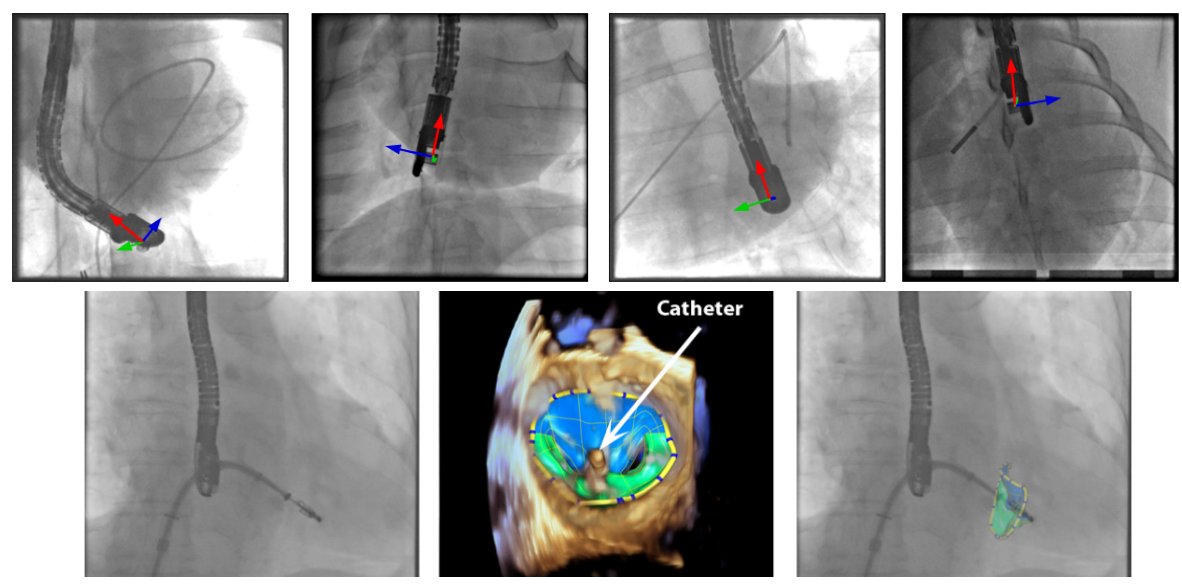

Fig. 4. Top - Fluoro images showing the detected pose of probe. Bottom: Left- Fluoro image. Center - mitral valve detected in 3D TEE. Right - Valve model visualized in Fluoro.

The out-of-plane $(\theta r, \theta p)$ detectors are analyzed on the synthetic data to evaluate the accuracy of the binary template matching. Fig. 3 plots the $(\theta r, \theta p)$ error over the search space (degrees) and illustrates stable detection with a single outlier.

The framework is evaluated with respect to all parameters (Table 2). Quantitative validation was performed on synthetic and phantom data (in vivo ground truth data was not available). The largest error is in the $\mathrm{Z}$ axis, which corresponds to the optical axis of the Fluoro device. It is expected that this is the largest error because estimating distance along the optical axis is challenging from a monocular Fluoro image. Fortunately, the goal of the framework is to visualize anatomy in the Fluoro image, therefore errors in $\mathrm{Z}$ has little effect on the final visualization. Initial clinical feedback suggests errors of up to $15^{\circ}$ and $10 \mathrm{~mm}$ (excluding $Z$ ) are acceptable for some visualizations, however accuracy requirements are application specific. Qualitative evaluation (Fig. 4 top) is performed on in vivo Fluoro images.

Table 2. Quantitative validation of TEE probe detection

\begin{tabular}{ccccccc}
\hline Data & $\mathrm{X}(\mathrm{mm})$ & $\mathrm{Y}(\mathrm{mm})$ & $\mathrm{Z}(\mathrm{mm})$ & $(\theta r)$ & $(\theta p)$ & $(\theta y)$ \\
\hline Synthetic & $0.82(0.79)$ & $0.97(2.1)$ & $64.0(13.9)$ & $4.2(10.5)$ & $4.6(9.0)$ & $2.6(3.2)$ \\
Phantom & $1.1(0.8)$ & $0.7(0.6)$ & $19.04(1.6)$ & $11.5(12.0)$ & $11.8(9.8)$ & $3.0(3.4)$ \\
\hline
\end{tabular}

The computational performance was evaluated (Intel 2.13GHz single core, $3.4 \mathrm{~GB}$ RAM). The average detection time is 0.53 seconds. The computational cost can be reduced by incorporating temporal information to reduce the search space.

To illustrate the clinical relevance of this work an anatomical model of the mitral valve is detected [9] in 3D TEE and visualized in Fluoro (Fig. 4 bottom). The data is not synchronized and is manually fused. A catheter is visible in both modalities. 


\section{Conclusions}

This paper presents a novel method for automated fusion of TEE and Fluoro images to provide guidance for cardiac interventions. The proposed system detects the pose of a TEE probe in a Fluoro image. Discriminative learning is combined with fast binary template matching to address the challenges of pose detection. Validation has been performed on synthetic, phantom and in vivo data. The method is capable of detecting in $0.5 \mathrm{~s}$ with an in-plane accuracy of less than $5 \mathrm{~mm}$. Future work will focus on incorporating temporal information, using the initial detected pose as a starting estimate for pose refinement and visualization of anatomically meaningful information.

\section{References}

1. Jain, A., Gutierrez, L., Stanton, D.: 3D TEE Registration with X-Ray Fluoroscopy for Interventional Cardiac Applications. In: Ayache, N., Delingette, H., Sermesant, M. (eds.) FIMH 2009. LNCS, vol. 5528, pp. 321-329. Springer, Heidelberg (2009)

2. Ma, Y., Penney, G.P., Bos, D., Frissen, P., Rinaldi, C.A., Razavi, R., Rhode, K.S.: Hybrid echo and $\mathrm{X}$-ray image guidance for cardiac catheterization procedures by using a robotic arm: a feasibility study. Physics in Medicine and Biology 55, 371-382 (2010)

3. Gao, G., Penney, G., Ma, Y., Gogin, N., Cathier, P., Arujuna, A., Morton, G., Caulfield, D., Gill, J., Aldo Rinaldi, C., Hancock, J., Redwood, S., Thomas, M., Razavi, R., Gijsbers, G., Rhode, K.: Registration of 3D trans-esophageal echocardiography to X-ray fluoroscopy using image-based probe tracking. Medical Image Analysis 16, 38-49 (2012)

4. Gao, G., Penney, G., Gogin, N., Cathier, P., Arujuna, A., Wright, M., Caulfield, D., Rinaldi, A., Razavi, R., Rhode, K.: Rapid Image Registration of Three-Dimensional Transesophageal Echocardiography and X-ray Fluoroscopy for the Guidance of Cardiac Interventions. In: Navab, N., Jannin, P. (eds.) IPCAI 2010. LNCS, vol. 6135, pp. 124-134. Springer, Heidelberg (2010)

5. Lang, P., Seslija, P., Chu, M.W.A., Bainbridge, D., Guiraudon, G.M., Jones, D.L., Peters, T.M.: US-Fluoroscopy Registration for Transcatheter Aortic Valve Implantation. IEEE Transactions on Biomedical Engineering 59, 1444-1453 (2012)

6. Wu, W., Chen, T., Wang, P., Zhou, S.K., Comaniciu, D., Barbu, A., Strobel, N.: Learningbased hypothesis fusion for robust catheter tracking in 2D X-ray fluoroscopy. In: CVPR, pp. 1097-1104 (2011)

7. Hinterstoisser, S., Lepetit, V., Ilic, S., Fua, P., Navab, N.: Dominant orientation templates for real-time detection of texture-less objects. In: CVPR, pp. 2257-2264 (2010)

8. Taylor, S., Drummond, T.: Multiple Target Localisation at over 100 FPS. In: BMVC (2009)

9. Ionasec, R.I., Voigt, I., Georgescu, B., Wang, Y., Houle, H., Vega-Higuera, F., Navab, N., Comaniciu, D.: Patient-specific modeling and quantification of the aortic and mitral valves from 4-D cardiac CT and TEE. IEEE Trans. on Med. Imag. 29, 1636-1651 (2010) 